\title{
Tecnología, materia y lugar: Procesos de moder- nización en la obra española de la posguerra. Instituto de enseñanza media, Málaga. Arquitecto: Miguel Fisac
}

\author{
Technology, materials and space: Processes of modernization \\ of spanish post-civil war architecture. \\ High School, Málaga. Architect: Miguel Fisac
}

M. Loren ${ }^{(*)}$

RESUMEN

La trayectoria de Miguel Fisac (1913-2006) recorre el siglo XX de la arquitectura española, dejando traslucir la complejidad de la búsqueda de la modernidad en un régimen que reclamaba formas academicistas para su mensaje identitario nacional. El Instituto de Enseñanza Media y Escuela de Comercio en Málaga con sus bellos pórticos de hormigón de sección variable, su tratamiento científico de la luz, el espacio humanizado de sus patios, y la vinculación al lugar, ejemplifica una apuesta por la arquitectura como laboratorio tecnológico.

La documentación original nos desvela su valor añadido al tratarse de un segundo proyecto del autor; en este artículo se analiza la obra construida, en contraste con la monumentalidad y simetría del primer proyecto; representando los cambios operados en la arquitectura española de posguerra. Fisac introduce una nueva arquitectura docente en el paisaje andaluz de mediados del XX. Profundamente deteriorada, esta investigación propone su puesta en valor.

109-39

Palabras clave: Fisac; arquitectura española posguerra; arquitectura moderna en Andalucía; arquitectura docente; instituto Málaga.

\section{SUMMARY}

Miguel Fisac's career spans the twentieth century Spanish architecture (b.1913d.2006) and mirrors the complexity characterising the search for modernity in a regime concerned with reclaiming an academicism in order to convey the clichés of nationalism. Challenging official architecture, the High School and School of Commerce in Malaga, with its succession of concrete porticoes, his technical use of light and the humanized space of its patios, exemplifies this stake in modern architecture as a field for technological experimentation.

The documentation discloses an added value to this work, revealing that there had been two proposals. This article compares these, contrasting the actual building to the monumentality and the symmetry of the first proposal, corroborating a major shift that took place in Spanish post-war architecture. Fisac introduced a new educational architecture into the Andalusian landscape of the midtwentieth century. Deeply deteriorated, this research seeks to establish its value by means of a rigorous process of documentation.

Keywords: Fisac; Spanish post-civil war architecture; Modern architecture in Andalusia; educational architecture; high school Málaga.

(*) ETSA, Universidad de Sevilla, Sevilla (España) Persona de contacto/Corresponding author: marloren@us.es (M. Loren-Méndez)
Recibido/Received: 05 oct 2010 Aceptado/Accepted: 25 oct 201 Publicado online/ Published online: 05 may 2012 
1. Portada de la revista Nueva Forma, n. 39 (abril 1969) con la galería Noroeste Sureste que vertebra el Instituto de Enseñanza Media, la Escuela de Comercio y los espacios comunes de Capilla y Salón de Actos.

2. Proyecto de emplazamiento de edificios escolares en "El Egido", Málaga. Estado Actual. Escala 1:500, marzo 1944, Miguel Fisac y Ricardo Fernández Vallespín. Primer proyecto de 1944 del El Egido que incluye Instituto de Enseñanza Media, la Escuela de Comercio y Escuela de Trabajo en torno a una plaza.

Legado Fisac, facilitadas por la Fundación Fisac, Ciudad Real.

\section{EJES Y GESTOS DE UN RÉGIMEN. PRIMER PROYECTO: EL EGIDO 1944-1948}

A través de su ondulante, compleja y dilatadísima gestión profesional, pueden traslucirse todos los avatares, incidencias, perplejidades y revelaciones del premioso, intermitente discurso arquitectónico de los últimos treinta años (1).

La contundente trayectoria de Miguel Fisac (1913-2006) recorre el siglo XX de la arquitectura española, dejando traslucir sin pudor la complejidad que marcó la difícil búsqueda de la modernidad en un país atracado en un régimen dictatorial que reclamaba los volúmenes compactos y las rotundas simetrías academicistas para asegurar la obviedad de su mensaje identitario nacional. En el período comprendido entre 1950 y 1958, en los años germinales como los denominaría Vicente PatónAlberto Tellería, se produce un abandono de las comprensibles incertidumbres de su primeros años de profesión, tranformando el convencionalismo en racionalidad creativa (2). Su producción es ingente, y la cualidad arquitectónica fundamentada en un compromiso con la innovación tecnologíca aparece en estos años cincuenta para quedarse.

El proyecto del autor para Instituto de Enseñanza Media y Escuela de Comercio (actual Instituto de Enseñanza Secundaria Ntra. Sra. de la Victoria y Escuela de Enfermería) en la ciudad de Málaga es especialmente representativo de los cambios operados en España en la producción arquitectónica de los años cuarenta y cincuenta del pasado siglo. Las dos soluciones tan distintas que el arquitecto desarrolla por encargo del Ministerio de Educación Nacional (1944-48 y 195364) representan nítidamente su actitud de búsqueda en estos difíciles años de la autarquía, del abandono paulatino del monumentalismo de ecos italianos y de un agolpamiento instantáneo del énfasis en lo constructivo, en la búsqueda de las capacidades expresivas de los materiales, en una modernidad fundamentada en la concepción arquitectónica desde la expermientación tecnológica y la interpretación de las condiciones del lugar.

En 1969 la reiteración estandarizada de sus pórticos de hormigón de sección variable que definieran los espacios de cobijo y encuentro para sus usuarios servía de portada al número 39 de la revista Nueva Forma en abril de 1969, definiendo como "Años Experimentales" la producción arquitectónica de Miguel Fisac en este período (Figura 1).

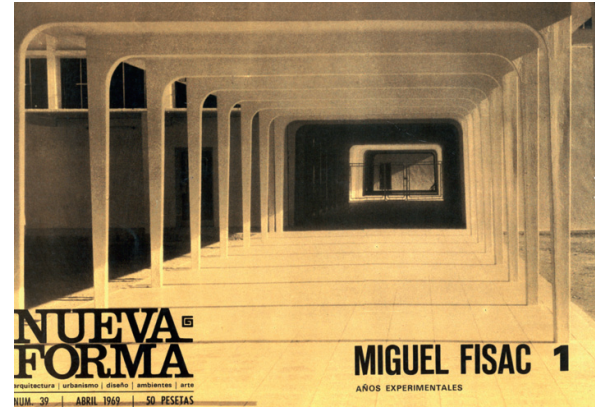

El descubrimiento y estudio de su primer proyecto para este programa en la ciudad de Málaga le añade si cabe un valor añadido: junto con la innovación técnica, el descubrimiento del espacio como materia principal de la arquitectura y la incorporación de los parámetros del lugar, desvelan la capacidad de actualización continua del autor. El primer proyecto realizado por Fisac junto con Ricardo Fernández Vallespín está fechado en 1944 y entregado al Ministerio de Educación Nacional en 1948, y por tanto enmarcado en su primeros años de oficio, tras haber terminado la carrera en 1942.

El encargo contempla la construcción de edificios de Instituto de Enseñanza Media, Escuela de Comercio y Escuela de trabajo. Este primer encargo se localiza en la zona denominada "El Egido", en un solar de 56.881 metros cuadrados (3). Los arquitectos proponen una composición axial inequívocamente académica en la que el edificio del instituto ocupa el lugar central, estableciendo el primer límite de una plaza elevada sobre pedestal conformando la escuela de comercio y la escuela de trabajo los frentes laterales de un esquema en $U$ (Figura 2).

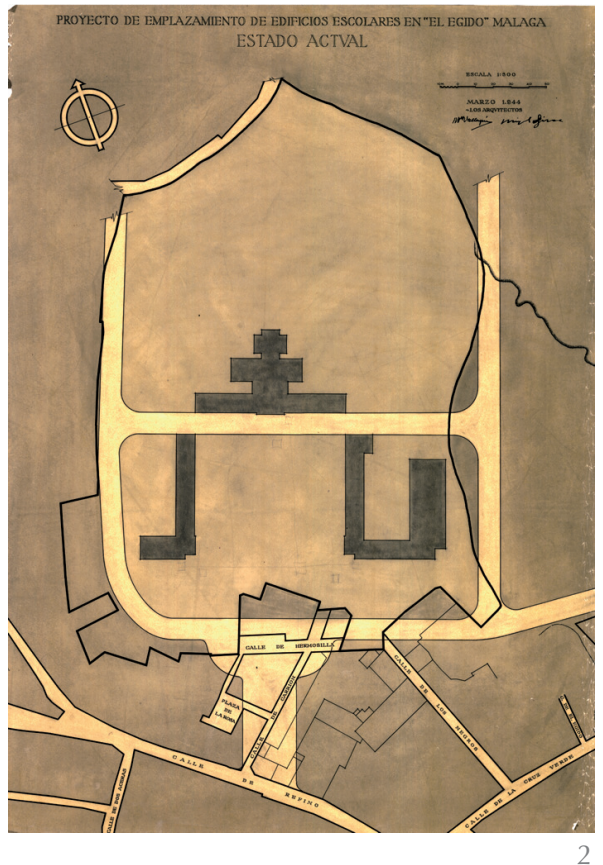


La insistencia en un entendimiento monumental trasciende a la escala paisajística y urbana: por un lado, Fisac apunta sobre el carácter elevado del solar, siendo visible incluso desde el mar, permitiendo así construir un icono para la ciudad. Por otro, y aprovechando el compromiso adquirido por el Ayuntamiento de Málaga de realizar las obras de urbanización necesarias para conectar el solar con el centro de la ciudad, el conjunto está pensado como remate de la prolongación de la Calle Larios, -vía principal trazada en el XIX que vertebra el centro histórico, convirtiéndose así en un espacio público de primer orden de la ciudad malagueña. En un plano de la ciudad de Málaga incluye el Egido y marca una distancia de 600 metros con la Plaza de la Constitución, y de 200 metros con el Teatro Cervantes (Figura 3). la plaza recoge las aspiraciones ideales y estables de las representaciones renacentistas. Su tratamiento elevado, a modo de pedestal rematado con un muro de carácter defensivo confiere a la enseñanza un componente sacro, tan presente en la obra del autor y esencial en la construcción de una imagen nacional.

El proyecto opta por la gran dimensión tanto en la escala de los edificios como en el espacio no construido de la plaza. El uso de la abstracción en la geometría de sus huecos recuerda a un cuadro de Giorgio de Chirico, primando sin embargo la opción academicista y monumental de la propuesta de Fisac en la línea del Edificio Central del Consejo Superior de Investigaciones Científicas, realizado en 1943.

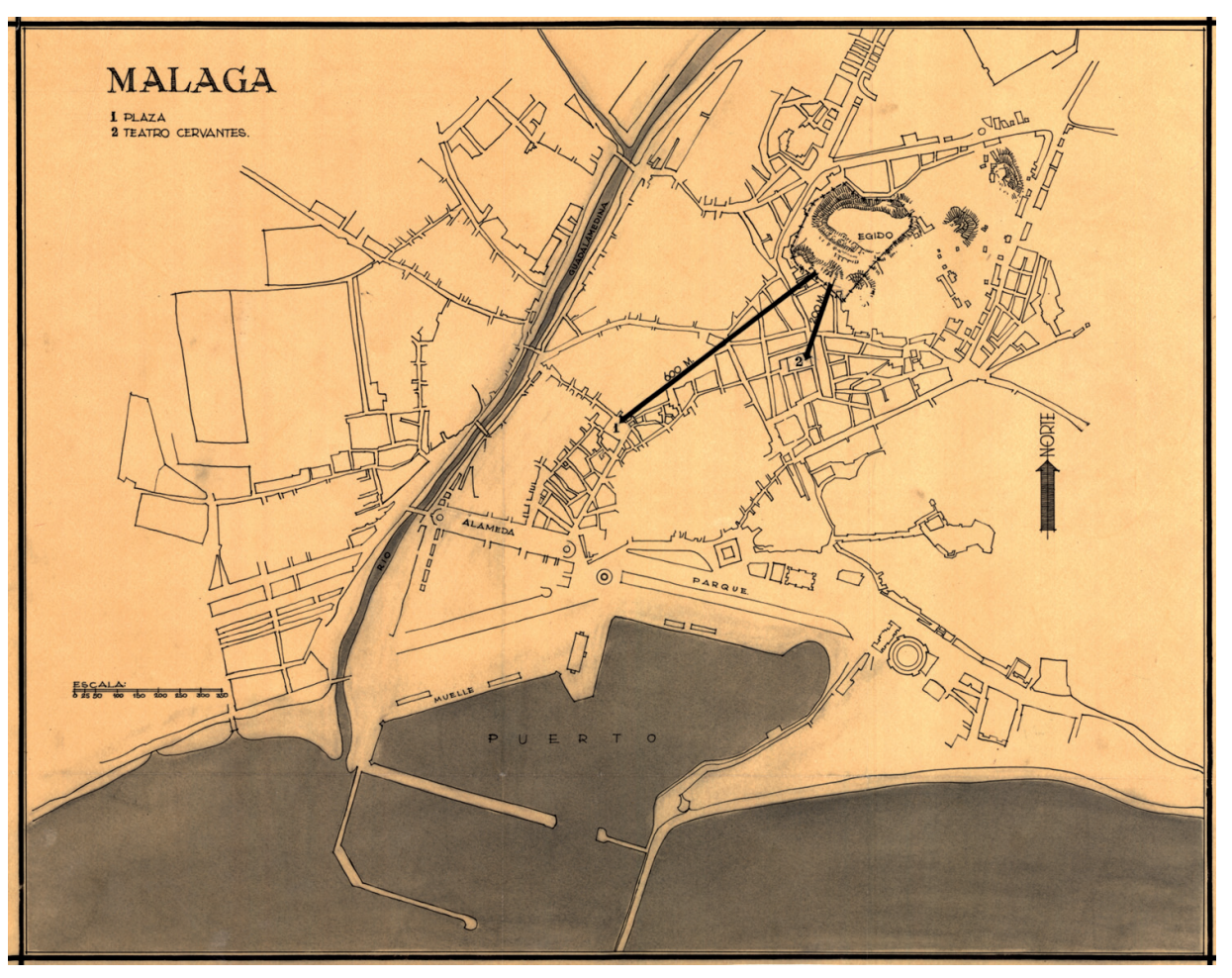

La cota del solar es algo más elevada que el resto de la población, dominándose actualmente, a pie, el mar por encima de la zona edificada más importante de Málaga. Al elevarse las edificaciones el panorama que desde ellas se ha de observar es espléndido, y desde el mar estos edificios forman un conjunto perfectamente destacado.

...Se ha determinado un eje principal que será también eje de una gran plaza encuadrada por los tres edificios que se proyectan y que es a su vez eje de la prolongación proyectada de la calle Larios (3).

La monumentalidad del conjunto queda así mismo fijada por la escala humana;
El mismo Fisac confirma que en esos años de 1943-1944 pensaba que el buen camino pueda ser tomar la modernidad cimentada sobre canónes clásicos: eternos. (4) En el diseño de sus fachadas corroboramos que esa cita ineludible a la arquitectura clásica y las obligadas referencias vernaculares en la volumetría tejada de su capilla, se encuentra ya en estas primeras obras de Fisac vinculada a una explícita austeridad ornamental y claridad volumétrica, a una tendente estandarización económica de la obra y expresión constructiva, mostrando el proyecto sus indicios de modernidad (Figuras 4,5 y 6 ).

El compromiso con la actualización constante de la arquitectura comenzaba así
3. Localización del solar de El Egido con relación a la ciudad de Málaga, distancias y posibles conexiones, Agosto 1944.

Legado Fisac, facilitadas por la Fundación Fisac, Ciudad Real. 


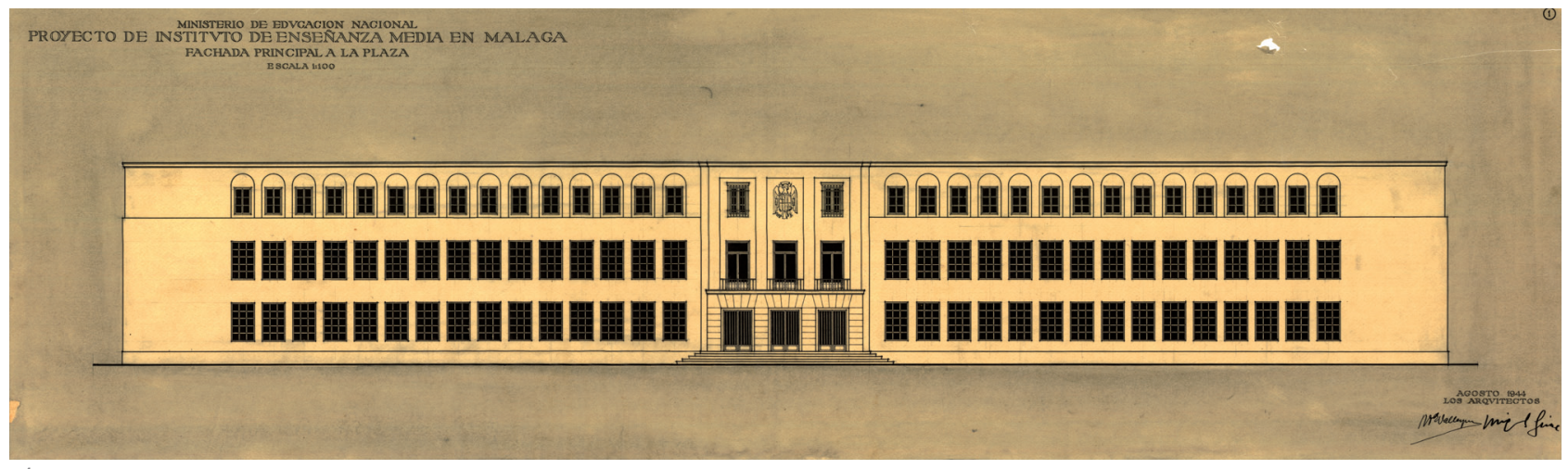

4
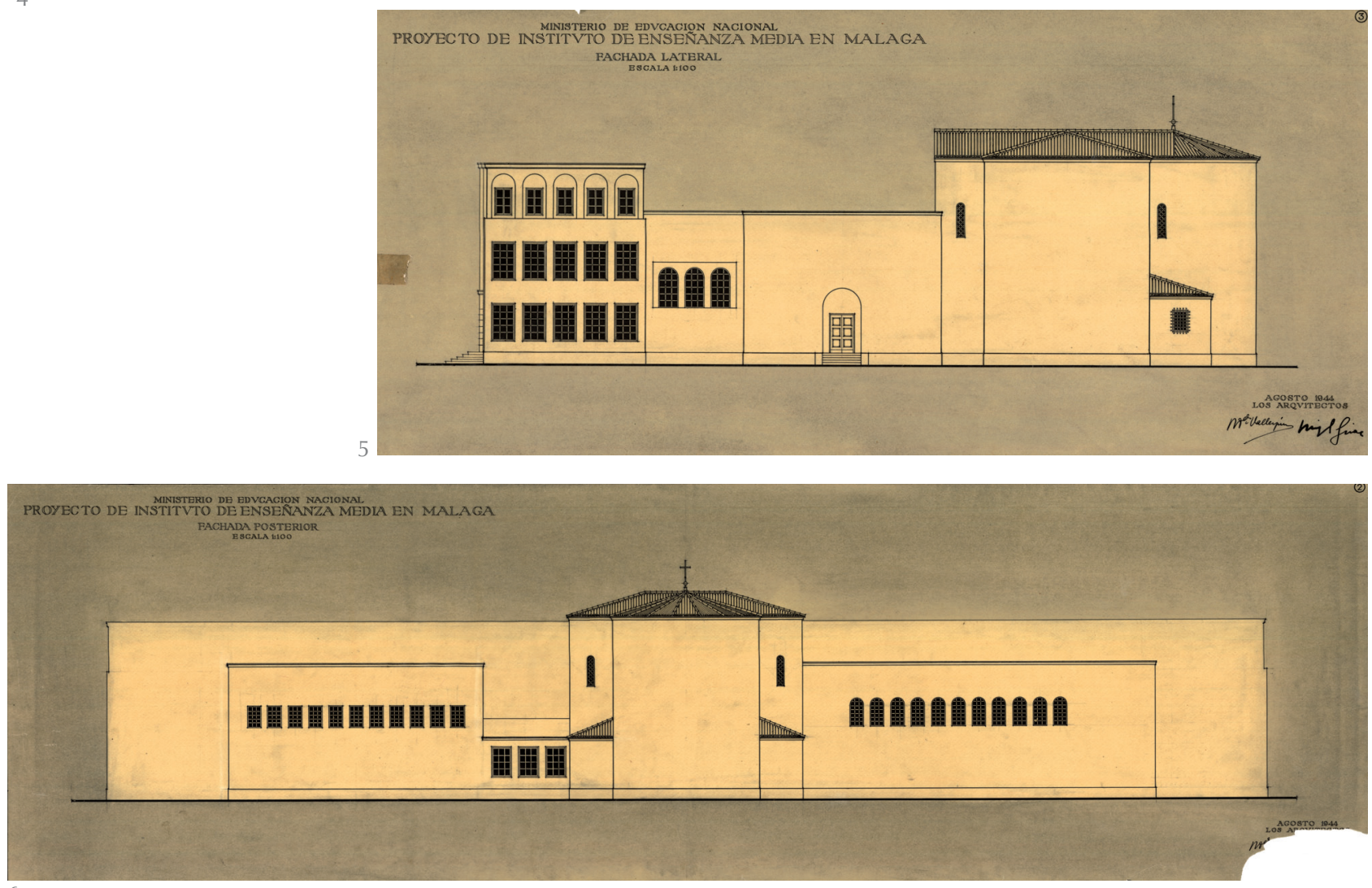
6

mismo a estar enunciada en estas obras de juventud también desde su propuesta programática y constructiva: en la memoria específica del Instituto de Enseñanza Media -fechada en julio de 1948- ya contemplaba el Museo de Ciencias Naturales, que apuntaba al componente moderno del encargo desde sus inicios $(5,6)$ junto con laboratorios, gabinetes de experimentación, gimnasio y observatorio astronómico meteorológico a los usos más convencionales de un instituto de enseñanza media. Su disposición en planta sin embargo, redundaba en el eje de simetría, con una primera pieza para las aulas y una segunda en la que un gran vestíbulo cuadrado repetía el esquema en $U$ con el gimnasio y el auditorio en los laterales y la capilla al fondo del eje de toda la composición desde Calle Larios, integrando a la perfección el mensaje del régimen (Figuras 7 y 8 ).
Desde el punto de vista constructivo propone una estructura de hormigón armado sobre pozos, dado el carácter arcilloso de los terrenos sobre los que se asienta. El sistema de forjado, definido en la memoria de 1948- es del tipo de suelo nervado, con bloques huecos de cemento, con una separación de 0,60 metros entre ejes de nervios y una altura total de 0,20 (canto útil de $18 \mathrm{~cm}$ ). La obra se estima en $18 \mathrm{me}-$ ses y 6.209.233,52 pesetas. Estas primeras obras, y a pesar de su apuesta formal clasicista, son consideradas en su momento de vanguardia -tal y como el mismo Fisac nos refiere sorprendido en su texto de Quaderns- éxito que sin embargo no impidió un sentimiento de decepción en el autor.

En la memoria para el segundo proyecto Fisac traza los antecedentes del proyecto elaborado entre 1944 y 1948 y afirma que el Ministerio de Educación Nacional había 
resuelto dedicar los terrenos de El Egido a otro tipo de edificaciones. Sin embargo, el edificio proyectado para Escuela de Trabajo se llega a construir, dirigiendo la obra el Aparejador Mariano Jaúregui y siendo dedicada al final a albergar parte de la Escuela Politécnica "Escuelas Técnicas de Peritos Industriales y de Maestría Industrial", inaugurándose en abril de 1961 por el General Francisco Franco $(7,8)$. Desde octubre de 1990 acogería la Escuela Técnica Superior de Ingeniería Industrial hasta su traslado al nuevo edificio en el Campus de Teatinos.

\section{PROYECTO CONSTRUIDO. INNOVACIÓN TECNOLÓGICA, EL LUGAR Y EL ESPACIO. MARTIRICOS 1953-1954 / 1961-1964}

\subsection{El cambio de rumbo}

"De una parte el nuevo emplazamiento y de otra el tiempo transcurrido, hace que sobre el conocimiento y construcción de edificios escolares el arquitecto que suscribe tenga un distinto concepto al del que tuvo cuando redactó el anterior proyecto, causa por la que ha considerado beneficioso el redactar un nuevo proyecto..." (9).

El retraso acumulado en los inicios del proyecto así como la decisión por parte del Ministerio de Educación Nacional de un cambio de ubicación se traducen en una metamorfosis profunda del proyecto; él mismo explica que el tiempo transcurrido, el cambio de solar y los nuevos conocimientos que él ha desarrollado en centros docentes desembocarían en un concepto distinto. Simetría, compacidad, clasicismo que son drásticamente descartados en el proyecto definitivo fechado en 1953. Proyectos como el del Conjunto de Teologado San Pedro Mártir de los Padres Dominicos en Madrid del año 1955 confirman la continuidad en esta línea proyectual del autor. El cambio supone, desde el punto de vista histórico, el testimonio de una maduración en la arquitectura de Miguel Fisac en un corto periodo de tiempo y en un mismo proyecto, hecho que incrementa su valor patrimonial no sólo en el marco de la producción del autor sino como paradígmatica de las transformaciones de la arquitectura española de posguerra. Su consideración en la historia de la arquitectura andaluza comienza con el estudio coordinado por el profesor Pérez Escolano de 1987 (10), a la que le sucederán una serie de publicaciones en las que se consolida la apreciación de esta obra de Miguel Fisac (11, 12, 13 y 14), aunque un estudio monográfico de la misma resulta hoy día inédito.

El proyecto del Instituto de Enseñanza Media y Escuela de Comercio, remitido por el
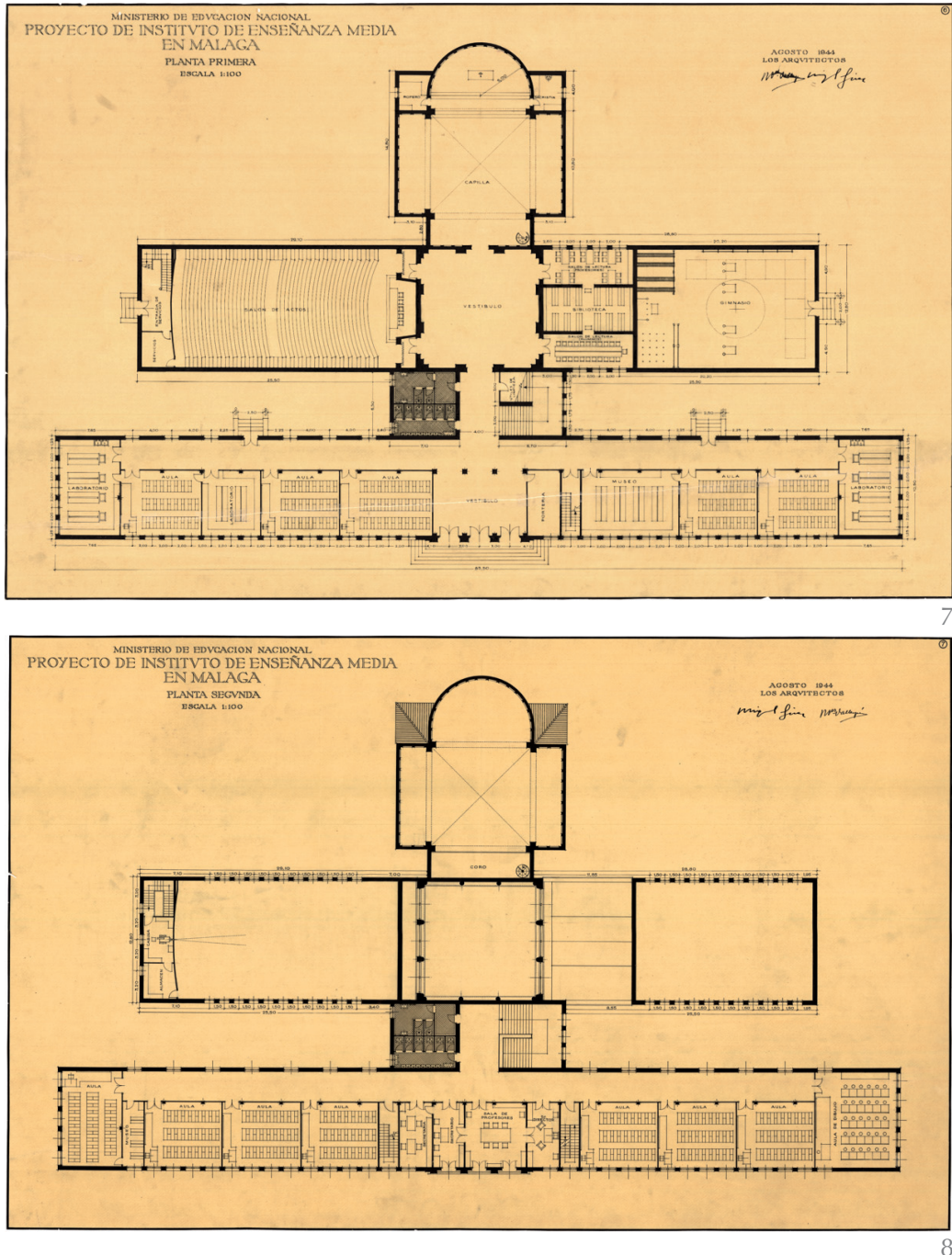

arquitecto Miguel Fisac Serna al Ministerio de Educación Nacional en Junio de 1953, se localizaba en un solar definido por cuatro calles con los accesos por el Paseo de los Martiricos, dos calles de nueva creación -actualmente la Avenida del Doctor Marañón y la Calle Toledo- y la Calle de los Ángeles, fachada que termina ocupando un grupo de viviendas reduciéndose a tres las calles que limitan el edificio.

El solar tiene una superficie de 28.250 metros cuadrados, de topografía sensiblemente horizontal. Su geometría es trapezoidal, siendo su lado mayor la fachada al Paseo de Matiricos, de orientación Oeste y por donde se plantean los accesos a ambos. La propuesta se dividía en dos proyectos, que Fisac definiría como tres etapas: Proyecto de Instituto de Enseñanza Media; Proyecto de Escuela de Comercio y por último Iglesia y Salón de Actos, como servicios comunes de ambas y que se incluiría en el proyecto de la Escuela de Comercio.

Centrándonos en el Instituto su programa comprende siete aulas, tres laboratorios, un aula de dibujo, el museo de historia natural 
9. Proyecto de Instituto de Enseñanza Media, Málaga. Plano de emplazamiento en el que se incluye también futuro proyecto de Escuela de Comercio y de zonas comunes con capilla y salón de actos, escala 1:500, junio 1953, Miguel Fisac.

Legado Fisac, facilitadas por la Fundación Fisac, Ciudad Real. arriba reseñado, biblioteca, gimnasio y zona de dirección -con sala de profesores, despachos de director y secretario- secretaría, sala de reunión de alumnos y portería. Fisac plantea en un primer momento la idoneidad de proyectar tanto el Instituto como la Escuela de Comercio en una sola planta, confirmando que no precisa de los mecanismos escalares en la aproximación al proyecto. Sin embargo, la importancia que adquieren las áreas libres obliga al autor a desarrollarlos en dos plantas.

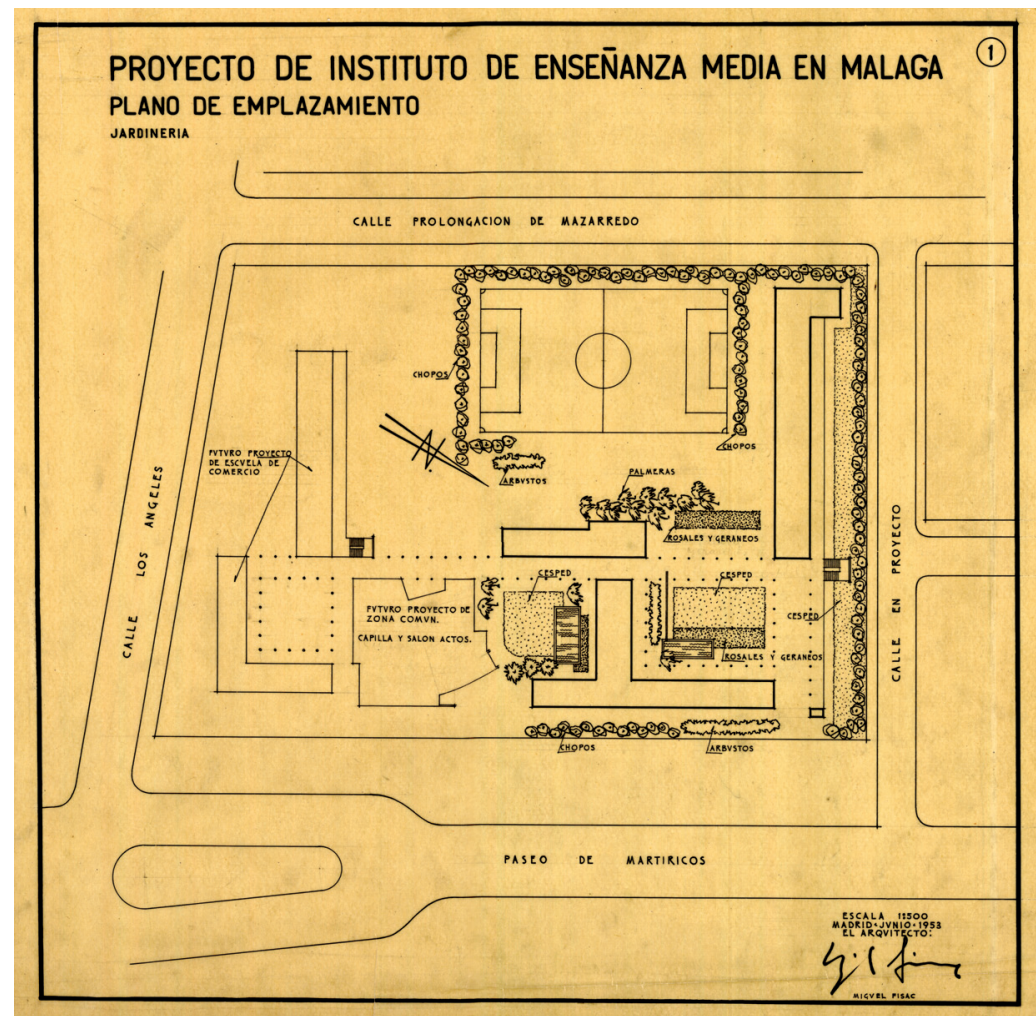

En la organización programática y volumétrica el arquitecto es explícito en el abandono de la línea proyectual desde la definición objetual, donde predominaba la lectura compacta de los distintos programas contenidos en edificios independientes y formando un conjunto reconocible en torno a una plaza monumental, calificando de anticuado y formalista la recurrencia a unos ejes, afirmando así mismo que la simetría es contraria a la función docente (Figura 9).

"La composición de las masas del edificio viene fijada por las orientaciones más convenientes de cada uno de los recintos de que está compuesto, dando lugar a una disposición compensada que sin ser ni mucho menos anárquica, ni responder a preocupaciones de lo que podríamos llamar un modernismo arquitectónico, huye también de ese otro anticuado y formal de unos ejes, de unas simetrías totalmente en disonancia con la función a que el edificio está destinado." (15)

Frente a la primera propuesta, en el proyecto construido el autor "disecciona" el volumen siguiendo el programa de usos, buscando para cada pieza construida su orientación más adecuada y su posición relativa con respecto a otras de las que depende, eliminando el concepto de fachada representativa y conjunto monumental y alejándose de las aspiraciones iconográficas a escala urbana y paisajística. El programa se distribuye así en distintas piezas longitudinales y espacios libres buscando su lógica en su funcionamiento cotidiano, planteando una escala casi doméstica para el aprendizaje (Figuras 10, 11 y 12).
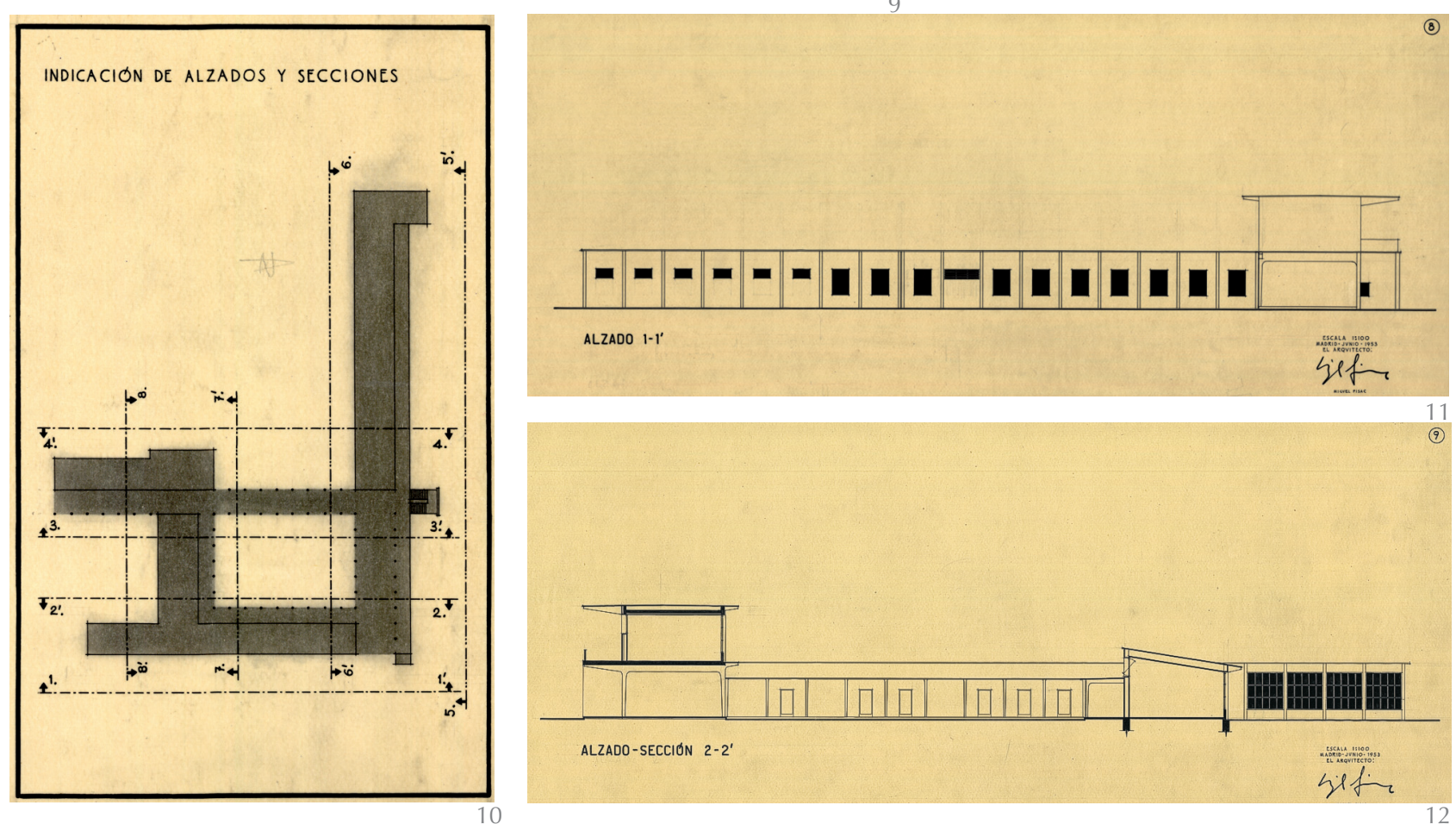


\subsection{La solución estructural como concepto de proyecto}

Fisac insiste en una concepción eminentemente espacial del proyecto arquitectónico, convirtiéndose el espacio no construido, y sus variaciones en elemento crítico del proyecto. El análisis de estas latitudes trasciende una orientación higienista de su programa construido; el proyecto plantea una gradación en la relación del usuario con el exterior. Aprovechando las óptimas condiciones climáticas de Málaga, las pastillas se estructuran a través de galerías de acceso abiertas (Figuras 13 y 14) y la transición entre las superficies abiertas y las distintas piezas construidas se realiza con grandes espacios abiertos pero cubiertos, entendiéndose estas galerías de acceso y comunicación como auténticas zonas de estancia (Figura 15). Los elementos estructurales que las cubren se convierten en el elemento formal que identifica el proyecto; sus bellos pórticos de hormigón con pilares de sección variable establecen una continuidad en el proyecto y vincula indefectiblemente las distintas piezas del proyecto, estableciendo una coherencia formal que se apoya en las capacidades del hormigón y sus posibilidades geométricas. (Figura 16).

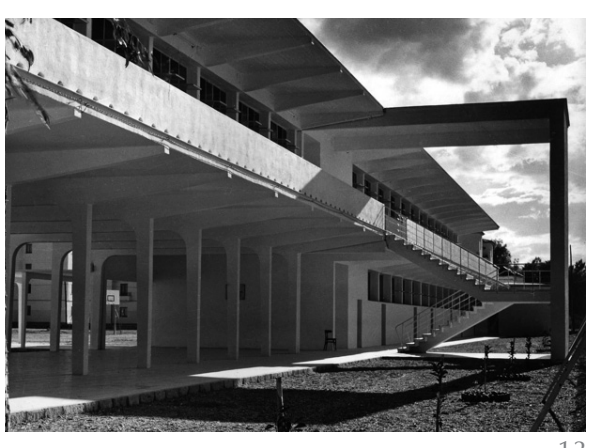

"Así como se ha considerado innecesario el cerrar las galerías de acceso, se considera, sin embargo, muy útil tener grandes superficies abiertas pero cubiertas, que protejan del Sol dando al conjunto una agradable variación de zonas abiertas, zonas abiertas pero cubiertas y recintos cerrados" (15).

La solución estructural trabaja con pórticos de hormigón de una luz de 5,95 metros y distancia entre ejes de 3,50 metros, con forjados de losa nervada con encofrado permanente. Las cubiertas son de terraza a la catalana, resuelta con baldosín catalán, cámara de aire y aislamiento de lana de vidrio. Las partes voladas que no son de terraza son de placa de hormigón impermeabilizada con productos bituminosos y fibra de vidrio. Las juntas de dilatación se resuelven con lámina metálica con membrana de lino y algodón e impermeabilizante bituminoso. La tectónica del hormigón domina el conjunto; pintados al silicato conservan el color y la textura del material.

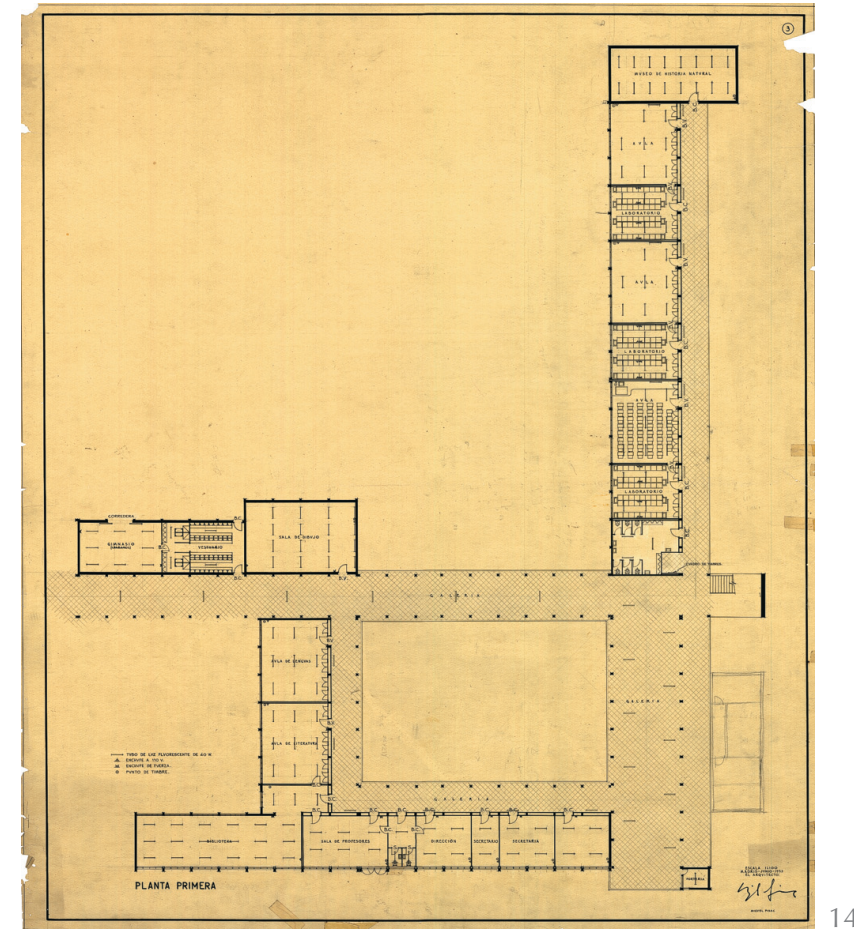

Los restantes cerramientos opacos van enfoscados y revocados con mortero de cemento. Todo ello corrobora el compromiso de este arquitecto con la explotación de la técnica en su práctica profesional. La innovación y singularidad de los elementos estructurales en estas arquitecturas de posguerra son ya en sí mismo de un valor patrimonial innegable.
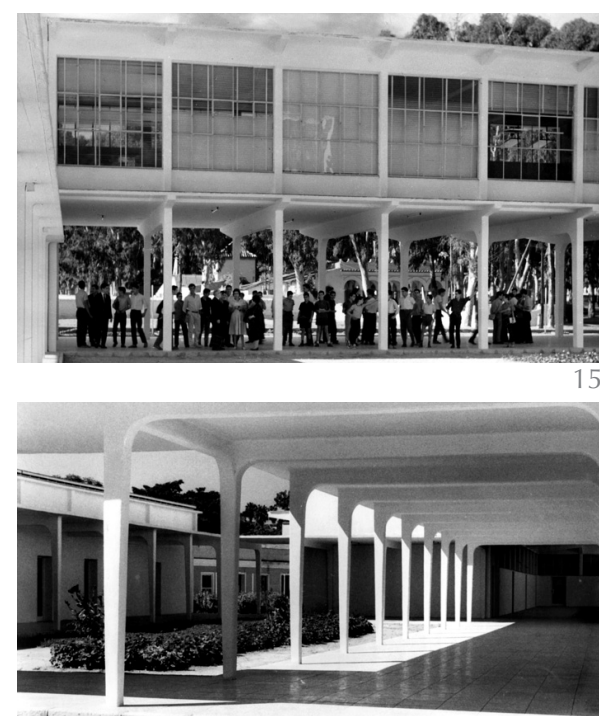

\subsection{Pensado desde el espacio que no se construye}

Los patios y zonas de juego se entienden as como extensión de dichos espacios cubiertos y abiertos, pero con la sutileza de gradación luminosa y térmica entre ambos espacios.

El tratamiento y escala de cada uno de estos ámbito se hace específico para cada parte
10. Proyecto de Instituto de Enseñanza Media, Málaga. Plano de indicación de fachadas y secciones, junio 1953.

Legado Fisac, facilitadas por la Fundación Fisac, Ciudad Real

11. Proyecto de Instituto de Enseñanza Media. Alzado 1-1', escala 1:100, junio 1953, Miguel Fisac. Legado Fisac, facilitadas por la Fundación Fisac, Ciudad Real.

12. Proyecto de Instituto de Enseñanza Media. Alzado-Sección 2-2', escala 1:100, junio 1953 , Miguel Fisac.

Legado Fisac facilitadas por la Fundación Fisac, Ciudad Real.

13. Proyecto de Instituto de Enseñanza Media. Vista de galería cubierta de acceso, con fachada norte de acceso a aulas y campos de juego al fondo.

Legado Fisac, facilitadas por la Fundación Fisac, Ciudad Real.

14. Proyecto de Instituto de En señanza Media. Planta primera, junio 1953, escala 1:500, Miguel Fisac.

Legado Fisac, facilitadas por la Fundación Fisac, Ciudad Real.

15. Proyecto de Instituto de Enseñanza Media, Málaga. Vista de galería cubierta de conexión con la Escuela de Comercio entendido como espacios de estancia. Legado Fisac, facilitadas por la Fundación Fisac, Ciudad Real.

16. Proyecto de Instituto de Enseñanza Media, Málaga. Vista de los distintos pórticos desde el patio.

Legado Fisac, facilitadas por la Fundación Fisac, Ciudad Real. 
17. Proyecto de Instituto de Enseñanza Media, Málaga. Vista del volumen de la capilla desde el patio.

Legado Fisac, facilitadas por la Fundación Fisac, Ciudad Real. del programa, matizando así los recorridos y la experiencia del edificio -en el caso del Instituto dos de ellos más pequeños ajardinados y el tercero que acoge los campos de juego y en el caso de la Escuela de Comercio un patio de acceso y distribución.

"Se ha procurado también, jugando con las orientaciones más convenientes de todas las partes del edificio, formar conjuntos gratos de patios, estudiando las diferentes jardinerías, estanques y surtidores, que sin plagiar las formas arquitectónicas locales, recogen el espíritu de lo que entendemos más acertado de ellas" (15).

La gran plaza clásica es sustituída así por el espacio menudo de los distintos patios, al cobijo sereno de sus desnudos pórticos blancos; sol y sombra, rincones y vegetación que hacen presente el lugar en el proyecto arquitectónico. Nos dice Fisac en la memoria que el edificio conecta con las formas arquitectónicas locales, aunque frente a la cita directa de la teja árabe del primer proyecto, éste no ha precisado de recursos directos para recordarnos que el proyecto está en un lugar. El autor confirma así su entendimiento de la arquitectura como "un trozo de aire humanizado y bellamente delimitado" basado en la definición del filósofo chino Lao Tsé que en el año 600 a. c. afirma que "...cuatro paredes y un techo no son arquitectura sino el aire que queda dentro" (16).

\subsection{El espacio sacro. Experimentación con el concepto de muro dinámico}

Pórticos y patios ayudan a coser el instituto de enseñanza media con la escuela de comercio, componiendo una única entidad proyectual continua que de hecho no se separa hasta épocas recientes cuando se produce el cambio de uso. Sus accesos son independientes y comparten el núcleo común de la capilla, convirtiéndose ésta en elemento iconográfico de la composición aunque sin ocupar el lugar central. Es la iglesia por tanto el único contenedor programático que es tratado desde lo objetual, adquiriendo su muro un carácter escultórico, y dejando patente el papel predominante de la religión en la educación del régimen y en el propia trayectoria vital de Miguel Fisac. Sin embargo, y aún siendo el volumen más destacado, su geometría y materiales utilizados consiguen una integración dentro del concepto fragmentario del proyecto (Figura 17).

El concepto de belleza en la arquitectura religiosa de Fisac no se vincula a elementos añadidos, superpuestos a la arquitectura -en esa distinción que Alberti hiciera entre belleza y ornamento- sino a la propia arquitectura, alcanzando esa belleza de la luz, el color y la forma:

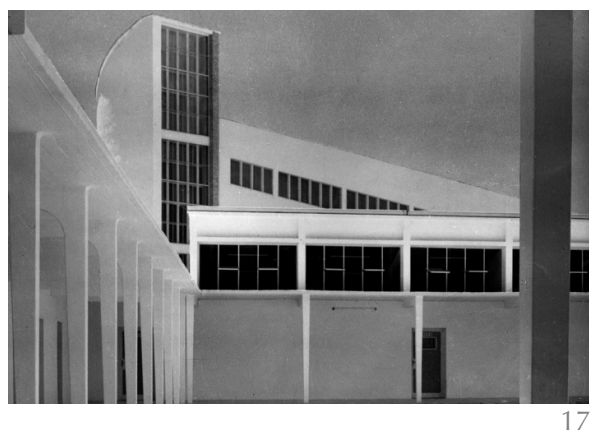

"En las iglesias fisacianas, la belleza no surge de elementos superpuestos a la arquitectura, sino que emana de la misma, de su propia estructura en conjunto y, por tanto, de sus propios elementos. ¿Y qué elementos son éstos? Para Fisac son: la luz, el color y la forma." (17)

En el proyecto de la capilla, insiste de nuevo en la importancia de la luz y del espacio, aunque esta vez especialmente comprometido con reproducir el carácter específico del templo católico, como un recinto para sus fieles que se congregan para la celebración litúrgica que converge y se mueve hacia el altar. Este proyecto de capiIla inaugura de hecho sus propuestas experimentales en torno al concepto de espacio dinámico que desarrollaría a lo largo de su carrera. Francisco Arques vincula así esta capilla de Ntra. Sra. de la Victoria del instituto malagueño a otras iglesias del autor como la Iglesia de Coronación en Vitoria. (18) Fisac se aleja de la artificiosidad en el diseño del espacio sacro; los elementos a utilizar, piensa el autor, deben mantenernos a distancia de la concepción maquinista y tecnológica del mundo contemporáneo, y por tanto deben ser austeros para hacer prevalecer su componente espiritual frente al material, sin dejar que la formalización sofisticada de sus límites eclipse la presencia del espacio, del espacio espiritual. El muro dinámico converge hacia el altar, quedando reducido su diseño a la luz, el color y la textura del ladrillo (Figuras 18, 19 y 20).

"... hemos de utilizar unos medios que podríamos llamar anti-fantásticos, sin trucos teatrales, de crudísima sencillez, que nos den, por contraste de la fantasía maquinista de la civilización que nos rodea, una posición real del hombre: su alma desnuda ante Dios" (19).

\subsection{Sistemas constructivos y orientaciones. Adecuación al uso docente: iluminación, ventilación y acondicionamiento acústico}

Las estancias son, así mismo, estudiadas desde la adecuación óptima a su uso y, por ende, la luminosidad necesaria y la 


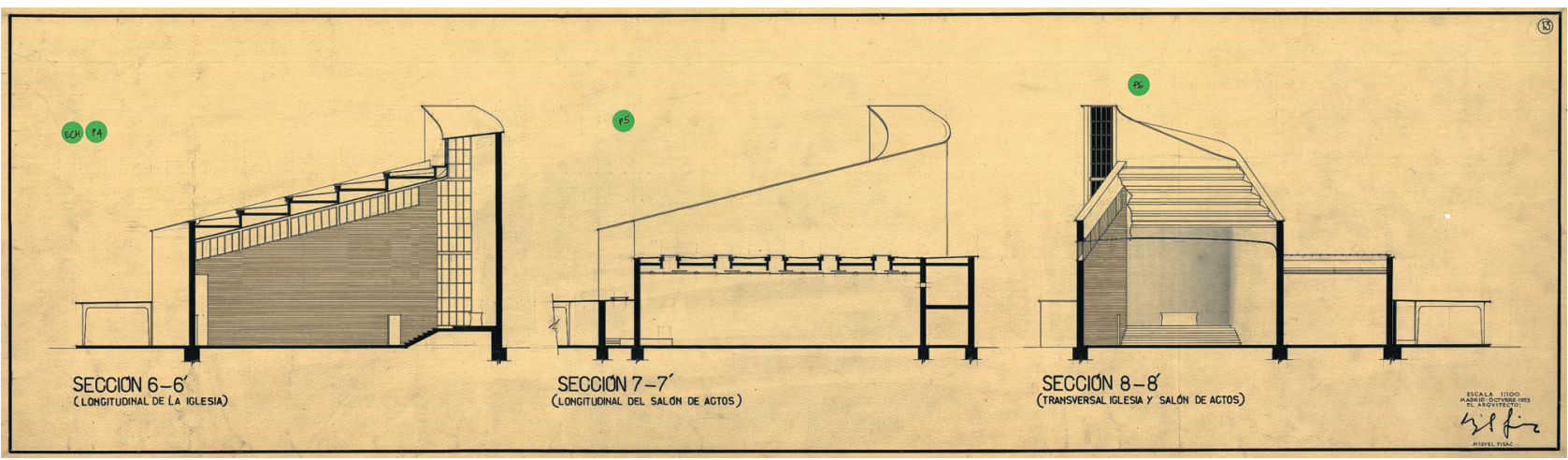

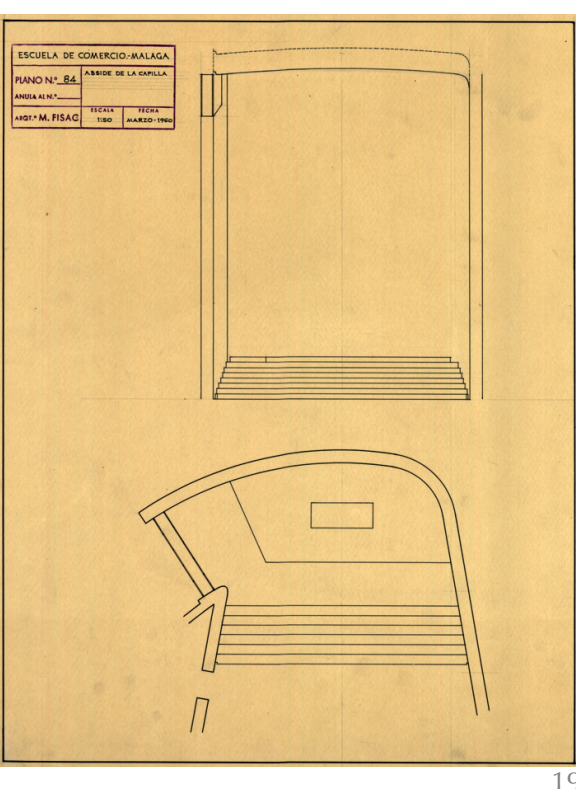

necesidad de una ventilación cruzada tanto en aulas como en laboratorios. Se han orientado hacia el sur por ser la orientación óptima de cara a la eficiencia energética, aunque para conseguir una iluminación uniforme y evitar la luz directa se recurre a incorporar una luz residual norte, desde la galeria abierta de acceso. Para conseguir una iluminación uniforme la fachada sur se resuelve con grandes superficies acristaladas con carpinterías metálicas (Figuras 21 y 22).

Para la protección solar Fisac descarta el uso de persianas enrollables, de uso extendido en los centros docentes, por

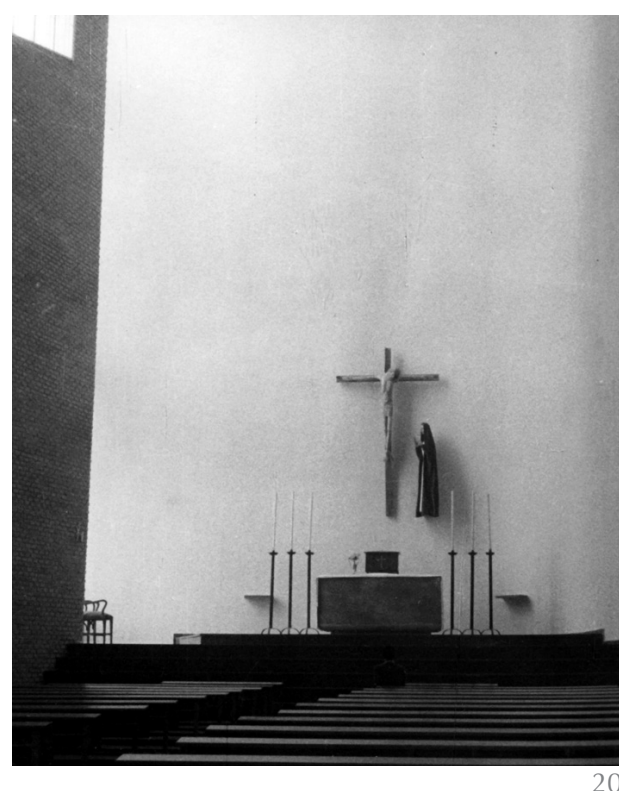

considerarlas poco prácticas y más aún en huecos de gran superficie. Opta así por persianas de aluminio tipo veneciano "Gradulux" pensadas tanto para la protección solar como para el oscurecimiento de las aulas. En el caso del aula de dibujo, la necesidad de la uniformidad lumínica es mayor recurriendo al sistema de dientes de sierra orientados al norte. Desde el patio de juegos el muro curvo y ascendente de la capilla es perceptible como fondo de las aulas de dibujo y lejos de aparecer diferenciado, pareciera que se trata de un diente de sierra que ha sido deformado para optimizar la captación de luz (Figura 23).
18. Proyecto de Instituto de Enseñanza Media. Secciones longitudinales -de la iglesia y del salón de actos- y sección transversal -iglesia y salón de actos, escala 1:100, junio 1953, Miguel Fisac. Legado Fisac facilitadas por la Fundación Fisac, Ciudad Real.

19. Proyecto de Instituto de Enseñanza Media, Málaga. Planta y sección del ábside de la capilla escala 1:50, marzo 1960, Miguel Fisac.

Legado Fisac facilitadas por la Fundación Fisac, Ciudad Real.

20. Proyecto de Instituto de Enseñanza Media, Málaga. Vista del interior de la capilla, hacia el ábside. Legado Fisac facilitadas por la Fundació Fisac, Ciudad Real.

21. Provecto de Instituto de Enseñanza Media, Málaga. Sección 8-8 donde se representan las grandes superficies acristaladas que caracterizan los módulos de aulas, escala 1:100, junio 1953, Miguel Fisac.

Legado Fisac, facilitadas por la Fundación Fisac, Ciudad Real.

22. Proyecto de Instituto de Enseñanza Media de 1953. Vista de la solución de fachada sur de las aulas con grandes superficies acristaladas con carpintería metálica durante la construcción. Legado Fisac, facilitadas por la Fundación Fisac, Ciudad Real.
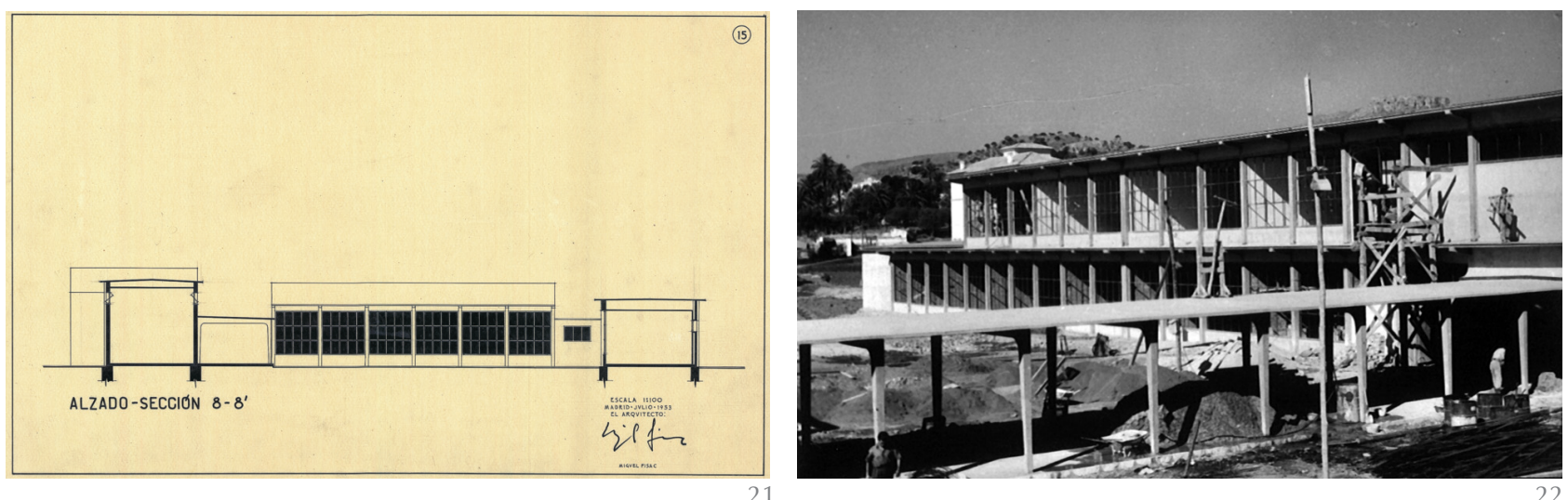
23. Proyecto de Instituto de Enseñanza Media, Málaga. Vista de los dientes de sierra con la capiIla al fondo.

Legado Fisac, facilitadas por la Fundación Fisac, Ciudad Real.

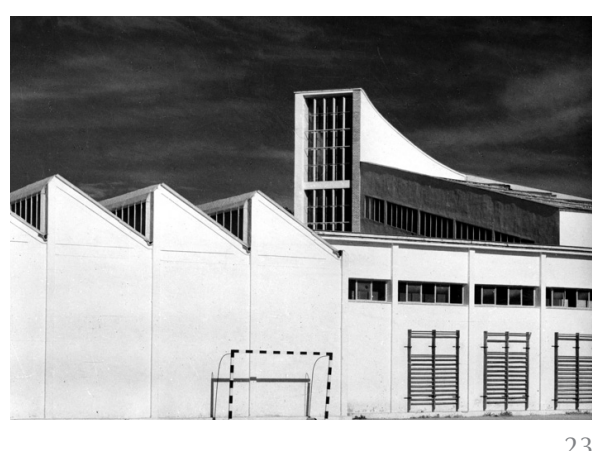

En las aulas también se tiene en cuenta cuestiones de aislamiento acústico, insonorización y oscurecimiento. El aislamiento acústico se realiza con doble tabique aislante entre las aulas y el acondicionamiento acústico se consigue colocando un techo insonoro a base de piezas táblex perforado. La pared frontal del profesor se acondiciona así mismo con revoco celulósico "Lovadi c.c."

\section{AMPLIACIONES DEL AUTOR}

En junio de 1961 Fisac redacta un primer proyecto de ampliación, consistente en 12 nuevas aulas -diez de 9 metros y dos de 6 metros- distribuídas en dos plantas, siguiendo el mismo criterio de proyecto que en la obra construida. Estas se ubican en la fachada este del campo de juego, por no existir otra ubicación posible para los mismos. Con el fin de resolver el acceso desde la pastilla de aulas del proyecto original, se demuele el extremo de contacto con la nueva construcción con el fin de incorporar las comunicaciones verticales y dar continuidad de ambas galerías. Se proyecta así mismo vivienda para conserje sobre Museo de Historia Natural. El tiempo de ejecución se estima en ocho meses y el presupuesto de contrata en 3.717.933,44 pesetas. (20) Esta obra la dirige con José Ramón Aspiazu Ordóñez, según consta en solicitud dirigida por los arquitectos al jefe de sección de edificios y obras del Ministerio de Educación Nacional, fechada el 7 de noviembre de 1963.

En enero de 1964 se presenta un segundo proyecto de ampliación para un nuevo pabellón que se localiza en la fachada norte del campo de juego, conectándose a la primera ampliación y separando definitivamente la Escuela de Comercio del Instituto. La segunda ampliación consta de un aula, dos despachos y servicios sanitarios de enfermería, despacho médico, sala de curas y cuarto de rayos $X$. Fisac explicita que el nuevo edificio procura conjugar perfectamente con el mismo criterio de ordenación y simplificación a los edificios ya construidos. El tiempo de ejecución se estima en seis meses y el presupuesto de contrata en $743.308,25$ pesetas. $(21)$

\section{EPÍLOGO}

En la actualidad la Escuela de Comercio es usada como Escuela de Enfermería y el Instituto sigue utilizándose como tal. La primera ha sido menos modificada, conservando el color blanco original en sus pórticos de hormigón y manteniendo escaleras y carpinterías metálicas originales aunque en mal estado. Las galerías en planta alta continúan abiertas, manteniéndose por tanto la cualidad espacial del proyecto original.

En el caso del Instituto, el color blanco ha sido cambiado por un amarillo ocre y un naranja teja, las carpinterías originales han sido completamente sustituidas por otras de menor tamaño y protegidas por rejas. Las galerías de acceso a las aulas de planta alta han sido sistemáticamente cerradas -eliminando por tanto la luz norte que entraba desde las mismas- cambiando radicalmente la fachada y el espíritu de la obra. La capilla ha sido también pintada de amarillo ocre y su interior transformado para albergar el uso de salón de actos del instituto. Los revestimientos de paramentos interiores, a base de zócalos de madera así como su mobiliario deterioran seriamente la concepción espacial propuesta por el autor en la que el espacio y su luz eran los únicos componentes del mismo. Por otro lado, el instituto y la enseñanza de enfermería han sido separados, quedando la capilla como parte únicamente del instituto y generando el límite entre ambos una separación de sus espacios y galerías que elimina la lógica del proyecto como una unidad continua y no como dos edificaciones diferenciadas.

El complejo docente que Miguel Fisac proyectó para la ciudad de Málaga ha permanecido olvidada para la mayoría de la comunidad arquitectónica y por ende minusvalorada por sus actuales usuarios; su estudio monográfico comenzó en el marco pionero del Registro Andaluz de Arquitectura Contemporenea, proyecto de investigación, documentación y difusión de la arquitectura del siglo XX que ha sido posible gracias al Instituto Andaluz de Patrimonio Histórico y la Dirección General de Bienes Culturales. Profundamente transformada y deteriorada, esta pieza arquitectónica que materializó una nueva aproximación a los espacios para la enseñanza se disuelve lentamente en el magma agresivo de la ciudad en constante cambio y queda a la espera de una decidida puesta en valor de lo que sin duda es parte imprescindible de nuestro patrimonio arquitectónico contemporáneo. 


\section{BIBLIOGRAFÍA}

(1) Fullaondo, J. D.: “Miguel Fisac. Los años experimentales". Nueva Forma, n. 39 (abril 1969), pp. 3-6, 3.

(2) Patón-Alberto Tellería, V.: "Los años germinales 1950-58", www.fundacionfisac.org, 4 agosto 2010.

(3) Fisac Serna, M.: Memoria General de Proyecto de Instituto de Enseñanza Media, Escuela de Trabajo y Escuela de Comercio, Madrid, 4 agosto 1944, pp. 1-2. Legado Fisac, Fundación Miguel Fisac, Ciudad Real. Inédito.

(4) Fisac Serna, M.: "Asplund en el recuerdo". Quaderns d'Arquitecture i Urbanisme, $n^{\circ} .147$ (1981), pp. 33-34.

(5) Coloma, I.; Palomares Samper, J. A.: "Museo de Ciencias de Málaga", Museos y colecciones públicas de Málaga, pp. 79-83, Universidad de Málaga, Málaga, 1997.

(6) Arjones Fernández, A.: "La arquitectura de Miguel Fisac, entorno de postulados historiográficos relativos a lo monumental y el monumento. A propósito de una obra de Miguel Fisac en Málaga". Boletín de Arte, n. 24 (2003), pp. 323-336.

(7) Escuela Universitaria Politécnica, Presentación e Historia, http://www.politecnica.uma. es/Informacion-general/historia.htm, 23 agosto 2010.

(8) España, M. C.: Dos décadas como pieza clave en el desarrollo industrial. Industriales cumple veinte años entre el recuerdo de El Ejido y la ilusión por su nuevo edificio, Sur. es (9 marzo 2010).

(9) Fisac Serna, M.: Memoria de Instituto de Enseñanza Media en Málaga, Madrid, junio 1953, p. 1. Legado Miguel Fisac, Fundación Miguel Fisac, Ciudad Real. Inédito.

(10) Pérez Escolano, V. et al.: 50 años de arquitectura en Andalucía: 1936-1986, Consejería de Obras Públicas y Transportes, Sevilla, 1987.

(11) Mosquera Adell, E.: La vanguardia imposible. Quince versiones de arquitectura contemporánea andaluza, Consejería de Obras Públicas y Transportes, Sevilla, 1990.

(12) García Vázquez, C.; Pico Valimaña, R. et al.: "Momo Andalucía. Arquitectura del Movimiento Moderno en Andalucía", 1925-1965. Consejería de Obras Públicas y Transportes, Sevilla, 1999.

(13) Candau, M. E. et al.: Málaga. Guía de Arquitectura, Consejería de Obras Públicas y Transportes, Sevilla, 2005.

(14) Coloma Martín, I.; Camacho, R.: Guía Histórico-Artística de Málaga, Editorial Arguval, Málaga, 2006.

(15) Fisac Serna, M.: "Memoria de Instituto de Enseñanza Media en Málaga", Madrid, junio 1953, op. cit. p. 2-3.

(16) Morales, F.: Arquitectura religiosa de Miguel Fisac, Librería Europa D.L., Madrid, 1960, citado en A. Morell, Miguel Fisac, El espacio dinámico, p. 41. Colegio Oficial de Arquitectos de Castilla-La Mancha, Guadalajara, 2005.

(17) Garcia, F.: "El simbolismo en las iglesias de Fisac". Informes de la Construcción, vol. 58, n. ${ }^{\circ} 503$ (julio-septiembre 2006), pp. 19-32, 22.

(18) Arques Soler, F.: Miguel Fisac, p. 124, Ediciones Pronaos, Madrid, 1996.

(19) Fisac Serna, M.: "Conversaciones con Alberto Morell en su estudio de Cerro del Aire", Madrid, 1993-97". Extraído de A. Morell. Miguel Fisac. El espacio dinámico. Op. cit. p. 41.

(20) Fisac Serna, M.: Proyecto de ampliación del Instituto de Enseñanza Media en Málaga. Memoria, Madrid, junio 1961, p. 1-2. Fundación Miguel Fisac, Ciudad Real. Inédito.

(21) Fisac Serna, M.: Proyecto de nuevo pabellón para el Instituto de Enseñanza Media en Málaga. Memoria, Madrid, enero 1964, pp. 1-2. Legado Fisac, Fundación Miguel Fisac, Ciudad Real. Inédito. 